\title{
A modular positive feedback-based gene amplifier
}

\author{
Goutam J Nistala ${ }^{1 \dagger}$, Kang Wu $^{2 \dagger}$, Christopher V Rao ${ }^{2^{*}}$, Kaustubh D Bhalerao ${ }^{1}$
}

\begin{abstract}
Background: Positive feedback is a common mechanism used in the regulation of many gene circuits as it can amplify the response to inducers and also generate binary outputs and hysteresis. In the context of electrical circuit design, positive feedback is often considered in the design of amplifiers. Similar approaches, therefore, may be used for the design of amplifiers in synthetic gene circuits with applications, for example, in cell-based sensors.

Results: We developed a modular positive feedback circuit that can function as a genetic signal amplifier, heightening the sensitivity to inducer signals as well as increasing maximum expression levels without the need for an external cofactor. The design utilizes a constitutively active, autoinducer-independent variant of the quorumsensing regulator LuxR. We experimentally tested the ability of the positive feedback module to separately amplify the output of a one-component tetracycline sensor and a two-component aspartate sensor. In each case, the positive feedback module amplified the response to the respective inducers, both with regards to the dynamic range and sensitivity.

Conclusions: The advantage of our design is that the actual feedback mechanism depends only on a single gene and does not require any other modulation. Furthermore, this circuit can amplify any transcriptional signal, not just one encoded within the circuit or tuned by an external inducer. As our design is modular, it can potentially be used as a component in the design of more complex synthetic gene circuits.
\end{abstract}

\section{Background}

Positive feedback is a common mechanism involved in the regulation of genetic circuits [1]. Any time a gene product has the capacity to enhance its own production, either directly or indirectly, the circuit is said to involve positive feedback. A number of behaviors can be attributed to positive feedback loops. The defining one is clearly amplification. More complex behaviors include bistability and hysteresis. In addition, positive feedback is an integral element in many oscillatory, pattern-formation, and intracellular polarization processes $[2,3]$.

In a number of synthetic biology applications, positive feedback has been used to design switches, oscillators, and amplifiers. Besckei and coworkers [4], for example, showed in yeast that a simple positive feedback loop could transform a graded response to an inducer into a binary one. Likewise, Kramer and Fussenegger [5]

\footnotetext{
* Correspondence: cvrao@illinois.edu

† Contributed equally

${ }^{2}$ Department of Chemical and Biomolecular Engineering, University of Illinois at Urbana-Champaign, 600 S Mathews Ave, Urbana, IL, 61801, USA
}

showed that positive feedback could be used to generate hysteresis with respect to an inducer in mammalian cells. Maeda and Sano [6] analyzed a synthetic positive feedback loop in E. coli and demonstrated that it could give rise to either a graded or hysteretic response depending on the specific configuration. In terms of building circuits, Ajo-Franklin and coworkers [7] demonstrated that positive feedback could be used to engineer memory into yeast cells. Stricker and coworkers [8], on the other hand, built a simple oscillator by coupling positive feedback with negative feedback. In work most closely related to the present study, Sayut and coworkers $[9,10]$ demonstrated that a positive feedback loop could make the transcriptional activity of the quorum-sensing regulator LuxR more sensitive to autoinducer. In these regards, their design is most closely related to how positive feedback is typically employed in electronic circuits, namely to amplify the response to a signal.

In this work, we constructed a modular genetic amplifier in Escherichia coli based on a constitutively active, 
autoinducer-independent variant of the quorum-sensing regulator LuxR from Vibrio fischeri [11]. Our goal was to develop a simple network component that could be coupled to any cell-based sensing system where the output involves the transcription of some gene. In these regards, we sought to engineer an "off the shelf" device that could be readily implemented in any gene circuit. To test the ability of this device to amplify a transcriptional output, we coupled our device to a one-component tetracycline sensor and a two-component aspartate sensor. In both cases, we found that our amplifier was able to increase the sensitivity to the input signal and intensify the output signal.

\section{Methods}

\section{Media, growth conditions, and bacterial strains}

All cultures experiments were performed in either Luria-Bertani (LB) broth (tryptone: $10 \mathrm{~g} / \mathrm{L}$, yeast extract: $5 \mathrm{~g} / \mathrm{L}$, and $\mathrm{NaCl}: 10 \mathrm{~g} / \mathrm{L}$ ) or $\mathrm{M} 9$ minimal media supplement with $0.4 \%$ glucose, $1 \mu \mathrm{g} / \mathrm{mL}$ thiamine, and $1 \mu \mathrm{g} /$ $\mathrm{mL}$ biotin. All experiments were performed at $37^{\circ} \mathrm{C}$ unless noted otherwise. Antibiotics were used at the following concentrations: ampicillin at $100 \mu \mathrm{g} / \mathrm{mL}$, chloramphenicol at $20 \mu \mathrm{g} / \mathrm{mL}$, and kanamycin at $40 \mu \mathrm{g} / \mathrm{mL}$. Primers were purchased from IDT Inc. (Coralville, IA). Restriction enzymes were purchased from New England Biolabs Inc. (Ipswitch, MA) and Fermentas Inc. (Glen Burnie, MD) and used according to the manufacturer's recommendations.

All cloning steps were performed in E. coli strain DH5 $\alpha$. Subsequent experiments involving anhydrotetracycline induction were conducted in $E$. coli strain GN100 (F- ilvG rfb-50 rph-1 $\Delta e n v Z::$ FRT attB $\lambda::\left[\mathrm{P}_{\mathrm{N} 25^{-}}\right.$ tetR $\left.l a c I^{\mathrm{q}} s p c R\right]$ ) and those involving aspartate induction were performed in GN101 ( $\mathrm{F}^{-}$ilvG rfb-50 rph-1 $\Delta e n v Z:$ : FRT). Strain GN100 was constructed first by P1vir transduction of the $\Delta e n v Z:: k a n$ insert from JW3367-3 (The E. coli Genetic Stock Center, CGSC\# 10509) into MG1655. The antibiotic cassette from the FRT-KanFRT insert was then removed by transformation of pCP20 into the strain and selection on ampicillin at $30^{\circ} \mathrm{C}$ [12]. Loss of the helper plasmid pCP20 was obtained by growth at $42^{\circ} \mathrm{C}$ under non-selective conditions on LB agar. Lastly, the chromosomally integrated TetR/LacI expression cassette from DH5 $\alpha$ Z1 [13] was moved into this strain by P1vir transduction, yielding GN100. Similarly, strain GN101 was constructed in an identical manner except that it does not harbor the TetR/LacI expression cassette from DH5 $\alpha$ Z1.

\section{Plasmids Construction}

Table 1 provides a list of the plasmids used in this study. The plasmid pPROTetE-Kan-p15A was made by swapping the ColE1 origin of pPROTet.E with the p15A
Table 1 Plasmids used in this study

\begin{tabular}{|c|c|c|}
\hline Plasmid & Relevant characteristic & Reference \\
\hline pTJ003 & bla $\mathrm{P}_{l p p}$-taz ori $\mathrm{p} 15 \mathrm{~A}$ & {$[33]$} \\
\hline pPROTet.E & $\mathrm{cm} \mathrm{P}_{\text {Lteto-1 }}$ ori ColE1 & Clontech \\
\hline PPROBE-GFP & kan GFP[tagless] ori p15A & {$[15]$} \\
\hline pZE12-luc & bla $\mathrm{P}_{\text {Llaco-1 }}$-luc ori ColE1 & [13] \\
\hline pZE21 & kan $\mathrm{P}_{\text {LtetO-1 }}$ ori ColE1 & {$[13]$} \\
\hline $\mathrm{pZS24}$ & kan $\mathrm{P}_{\text {laclara-1 }}$ ori pSC101 & [13] \\
\hline pluxRI & $\mathrm{cm}$ Plac/ara-1-luxR-luxl ori ColE1 & {$[14]$} \\
\hline pPROTetE-kan-p15A & kan $\mathrm{P}_{\text {Lteto-1 }}$ ori $\mathrm{p} 15 \mathrm{~A}$ & \\
\hline pGN3 & kan $\mathrm{P}_{\text {Lteto-1 }}$-luxR * ori p15A & \\
\hline pGN11 & kan $\mathrm{P}_{\text {Lteto-1 }}-\mathrm{lux} \Delta_{2-156}$ ori p15A & \\
\hline$\overline{\mathrm{pGN12}}$ & kan $\mathrm{P}_{\text {LtetO-1 }}$-luxR $\Delta_{2-162}$ ori p15A & \\
\hline pGN23 & $\mathrm{cm}$ Plux $_{\text {ox }}$ ori ColE1 & \\
\hline pGN62-Kan & kan $\mathrm{P}_{\text {OmpC }}-\mathrm{lux} \Delta_{2-162}$ ori p15A & \\
\hline pGN68 & $\begin{array}{l}\text { cm Plux -GFP [tagless]-luxR } \Delta_{2-162} \text { ori } \\
\text { ColE1 }\end{array}$ & \\
\hline pGN69 & $\mathrm{cm} \mathrm{P}_{\text {lux }}$-GFP[tagless] ori ColE1 & \\
\hline pPROTetE-amp & bla $\mathrm{P}_{\text {LtetO-1 }}$ ori ColE1 & \\
\hline pGN76 & bla $\mathrm{P}_{\text {Lteto-1 }}$-taz ori ColE1 & \\
\hline$\overline{\mathrm{pGN77}}$ & bla $\mathrm{P}_{\text {Lteto-1 }}$-taz ori $\mathrm{pSC} 101$ & \\
\hline
\end{tabular}

Plasmids are from this study unless noted otherwise.

origin from pZA34-luc using the restriction sites XbaI and SacI and by swapping the chloramphenicol resistance gene with the kanamycin resistance gene from pZE21 using the restriction sites XhoI and SacI. The plasmid pPROTetE-Amp was made by replacing the chloramphenicol resistance gene in pPROTet.E with the ampicillin resistance gene from pZE12 using the restriction sites XhoI and SacI.

The luxI-GFP transcriptional fusion was made first by PCR amplification of the luxI promoter using the plasmid pluxGFPuv [14] as the template with the primers KW134F (CAG ATA TCG ACG TCA GTC C) and KW134R2 (ATA GAA TTC TGC GTT TAT TCG ACT ATA AC). The resulting fragment was then cloned into the plasmid pPROTet.E using the restriction sites EcoRI and AatII, yielding the plasmid pGN23. The green fluorescent protein (GFP) was PCR amplified from pPROBE$g f p$ [tagless] [15] using primers GN10F (GGG GAA TTC ATA CGT ATT TAA ATC AGG AGT GGA AAT GAG TAA AGG AGA AGA ACT T) and GN10R (GGG GGA TCC TTA TTA TTT GTA TAG TTC ATC CA). The resulting fragment was then cloned into the EcoRI and BamHI restriction sites of the pGN23, yielding the plasmid pGN69.

The LuxR* (LuxR[A221V]) expression plasmids were constructed using two rounds of PCR. In the first round, the $\operatorname{luxR}$ gene was amplified with primers KW78F1 (AAC TTT ATA AGG AGG AAA AAC ATA 
TGA AAA ACA TAA ATG CCG AC) and KW078R (ACT GTC GAC TTA ATT TTT AAA GTA TGG GC) using pLuxRI [14] as the template. The resulting product was then used as a template for a second round of PCR this time using primers KW078F2 (TAT GAA TTC AAC TAA AGA TTA ACT TTA TAA GGA GGA AAA ACA) and KW078R. It was then digested with EcoRI and SalI and sub-cloned into the EcoRI and SalI cut-sites of pPROTetE-Kan-p15A. Enzymatic inverse PCR was used to introduce the Ala221Val (GCG- > GTG) point mutation in the $l u x R$ gene with primers KW079F (ATA GGT CTC TGT GCA AAT GAA ACT CAA TAC AAC) and KW079R (ATA GGT CTC TGC ACA TTG GTT AAA TGG AAA GTG A). The resulting $\mathrm{PCR}$ product was then digested with $\mathrm{Bsal}$ and ligated to obtain pGN3.

The $\operatorname{lux} R \Delta_{2-156}$ expression plasmid was also constructed using two rounds of PCR. The luxR gene was first amplified with primers KW112F (AAC TTT ATA AGG AGG AAA AAC ATA TGA ACA TAC CAT TAA TTG TTC C) and KW078R using pLuxRI as the template. The resulting PCR product was then amplified using primers KW078F2 and KW078R. It was then cloned into the EcoRI and SalI cut-sites of pPROTetEKan-p15A, yielding pGN11. Likewise, the $l u x R \Delta_{2-162}$ expression plasmid was made by amplifying the $l u x R$ gene with primers KW113F (CTT TAT AAG GAG GAA AAA CAT ATG CCT TCT CTA GTT GAT AAT TAT C) and KW078R using pLuxRI as the template. The resulting product was amplified again as before using primers KW078F2 and KW078R. The PCR product was then digested with EcoRI and SalI and subcloned into the EcoRI and SalI cut-sites of pPROTetEKan-p15A, yielding pGN12.

The positive-feedback module was constructed using two rounds of PCR. In the first round, the primers GN09F2 (AAC TAA AGA TTA ACT TTA TAA GGA GGA AAA ACA TAT GCC TTC TCT AGT TGA TAA T) and KW171R (AAT AGC GGC CGC TTA TTA ATT TTT AAA GTA TGG GC) were used to amplify the luxR $\Delta_{2-162}$ domain [16] using pLuxRI [14] as the template. The resulting PCR product was then used as template for a second round of PCR this time using primers GN09F (GGG GGA TCC AAC TAA AGA TTA ACT TTA TAA GGA GGA AAA ACA T) and KW171R (AAT AGC GGC CGC TTA TTA ATT TTT AAA GTA TGG GC). The resulting fragment was then digested with BamHI and NotI and sub-cloned into pGN69, yielding pGN68.

The aspartate positive feedback module was constructed first by amplifying the $\mathrm{P}_{\text {omp }}$ promoter (genomic region 2310762-2310962) using primers GN03F (GGG CTC GAG GTT CCC TTG CAT TTA CAT TTT) and GN05R (GGG GAA TTC TAA CTT TCA
TGT TAT TAA CCC). The PCR product was then digested with XhoI and EcoRI and sub-cloned into the respective sites of pPROTetE-Kan-p15A, thus replacing the native $\mathrm{P}_{\mathrm{LtetO}-1}$ promoter with the $\mathrm{P}_{\text {ompC }}$ promoter. The primers GN06F2 (GGG GTC GAC ATG CCT TCT CTA GTT GAT AA) and KW171R were used to amplify luxR $\Delta_{2-162}$ using pGN68 as the template. The resulting PCR product was digested with SalI and NotI and then sub-cloned into the respective sites of pPROTetE-Kan-p15A, yielding pGN62.

The aspartate sensor module was constructed first amplifying the $t a z$ gene from pTJ003 using the primers GN13F (GGG GAA TTC TTA AAG AGG AGA AAG GTA CCC ATG ATT AAC CGT ATC C) and GN12R (GGG GTC GAC TTA CCC TTC TTT TGT CGT GCC CT). The PCR product was then digested with EcoRI and SacI and cloned into the unique respective restriction sites, yielding pGN76. The ColE1 origin in pGN76 was then replaced with the pSC101 origin from the pZS24 plasmid using the restriction sites AvrII and SacI, yielding pGN77.

\section{Fluorescence Assays}

To measure fluorescent protein expression, cultures were first grown overnight and then subcultured to an $\mathrm{OD}_{600}$ of 0.05 in fresh media. The cultures were first allowed to grow to an $\mathrm{OD}_{600}$ of 0.20 , at which point the inducer was added. The cultures were then grown overnight prior to taking the measurements. $100 \mu \mathrm{L}$ of the culture was then transferred into a 96 well microplate, and the relative fluorescence and optical density at $600 \mathrm{~nm}\left(\mathrm{OD}_{600}\right)$ were measured using a Tecan Safire2 microplate reader. The fluorescence readings, given as relative fluorescence units (RFU), were normalized with the $\mathrm{OD}_{600}$ absorbance to account for cell density. All experiments were performed in triplicate with 95\% confidence intervals reported.

\section{Results and Discussion}

\section{Design of positive-feedback amplifier}

In order to construct a positive feedback circuit, we required a transcriptional activator that did not interfere with native gene regulation in $E$. coli. In addition, we required that the activator be constitutively active and not dependent on the addition of an exogenous inducer. Given these constraints, we chose the LuxR protein from Vibrio fischeri [11]. This protein, normally involved in quorum sensing and bioluminescence, activates the transcription of the luxIADCBE operon in response to acyl homoserine lactone (AHL). AHL binding stabilizes the LuxR dimer and, as a result, increases its ability to activate transcription [17-19].

While wild-type LuxR does not appear to interfere with native $E$. coli regulation, it still requires an 
exogenous inducer. However, a number of approaches exist for making constitutively active derivatives of LuxR and thus satisfying our design constraints. For example, an Ala221Val point mutation was previously found to constitutively activate LuxR [20]. The alanine at position 221 enables the $\mathrm{N}$-terminal signaling domain to inhibit the activity of the C-terminal, DNA-binding domain. Presumably, mutating this residue to a valine prevents the $\mathrm{N}$-terminal domain from interfering with DNA binding. Consistent with this model, deleting the Nterminal domain of LuxR was also found to yield a constitutively active variant $[21,22]$.

Based on these previous studies, we engineered three constitutively active variants of LuxR to test their suitability in designing an amplifier. The first, denoted by LuxR*, harbors the Ala221Val point mutation. The other two, denoted by $\operatorname{LuxR}_{\Delta 2-156}$ and $\operatorname{LuxR}_{\Delta 2-162}$ respectively, involved different $\mathrm{N}$-terminal deletions, where the subscript denotes the deleted fragment. To test the relative effectiveness of these three different constitutive LuxR variants, we determined how strongly they could activate expression from the $\mathrm{P}_{l u x I}$ promoter, using the green fluorescent protein (GFP) as our transcriptional readout. The results from these experiments are shown in Figure 1. All of the LuxR variants, including the wild-type control, were able to induce expression from the $\mathrm{P}_{\text {lux I }}$ promoter. Of the three, only LuxR $\mathrm{R}_{\Delta 2-162}$ was capable in our hands of enhancing transcription

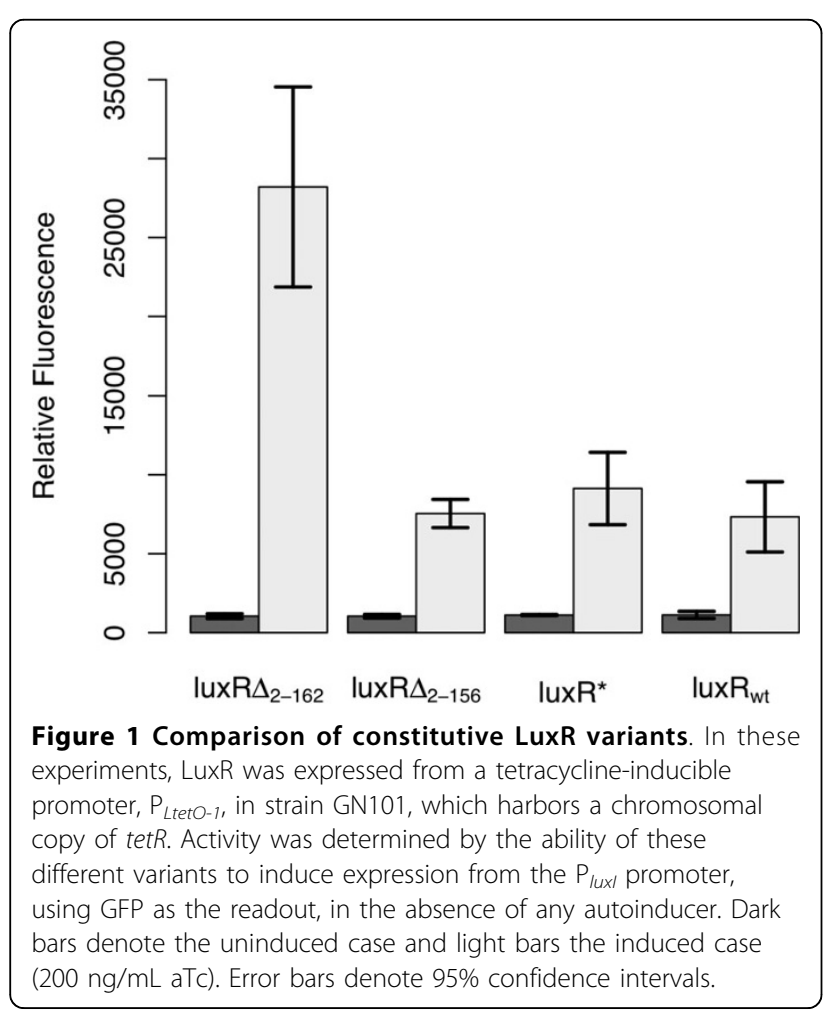

relative to the wild-type control. Based on these results, we chose to use the $\operatorname{LuxR}_{\Delta 2-162}$ variant to design the amplifier.

To construct the amplifier, we cloned GFP and LuxR $_{\Delta 2-162}$ in a bicistronic configuration behind the $\mathrm{P}_{\text {luxI }}$ promoter on high-copy number plasmid (ColE1 origin of replication). In this arrangement, $\operatorname{LuxR}_{\Delta 2-162}$ functions in a positive feedback loop as it can bind to the $\mathrm{P}_{l u x I}$ promoter and activate its own transcription (Figure 2). The reason we cloned $\operatorname{LuxR}_{\Delta 2-162}$ downstream of the GFP reporter is to control for polar effects when we compared results involving positive feedback to those lacking it. To induce this circuit, we again used $\operatorname{LuxR}_{\Delta 2-162}$, this time as the input signal. In such a design, the output of the sensor is $\operatorname{LuxR}_{\Delta 2-162}$, which in turn feeds back into the amplifier. In these regards, $\operatorname{LuxR}_{\Delta 2-162}$ is used both as the input and positive feedback signal. For the output, we used GFP as it provides a facile measure of transcriptional activity. This choice is in no way limiting, and any gene can in practice be used as the output.

\section{Validation of amplifier using a tetracycline sensor}

We first tested the amplifier by coupling it to a onecomponent tetracycline sensor (Figure 3). In this design, we cloned LuxR $\mathrm{R}_{\Delta 2-162}$ behind the TetR-regulated $\mathrm{P}_{\text {LtetO-1 }}$ promoter on a compatible, medium copy-number plasmid (p15A origin of replication) [13]. In the absence of the tetracycline analogue, anhydrotetracycline (aTc), dimeric TetR binds to the $\mathrm{O} 2$ operator sites within the $\mathrm{P}_{\text {LtetO-1 }}$ promoter and represses transcription. However, when TetR is bound with aTc, it no longer binds and represses the $\mathrm{P}_{\text {LtetO-1 }}$ promoter, enabling dose-dependent control of gene expression. Thus, the aTc-inducible promoter functions as a one-component tetracycline sensor with $\operatorname{LuxR}_{\Delta 2-162}$ as the output.

To couple this sensor with the amplifier, we transformed cells (GN100) constitutively expressing a

\section{LuxRA}

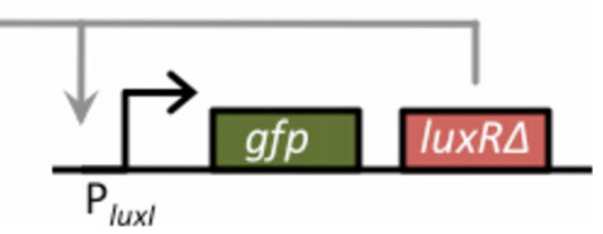

Figure 2 Schematic of positive-feedback amplifier. The basic design for the amplifier consists of GFP and LuxR $R_{\triangle 2-162}$ arranged in a bicistronic configuration under the control of the $P_{\text {luxl }}$ promoter. LuxR $R_{\Delta 2-162}$ functions in a positive feedback loop as it can bind to the $\mathrm{P}_{\text {luxl }}$ promoter and activate its own transcription. In our design, $\operatorname{LuxR}_{\triangle 2-162}$ is also used as the input signal for the amplifier. LuxR $R_{\triangle 2-}$ ${ }_{162}$, therefore, functions both as the input and positive feedback signal. GFP, the output signal, provides a measure of transcriptional activity. 


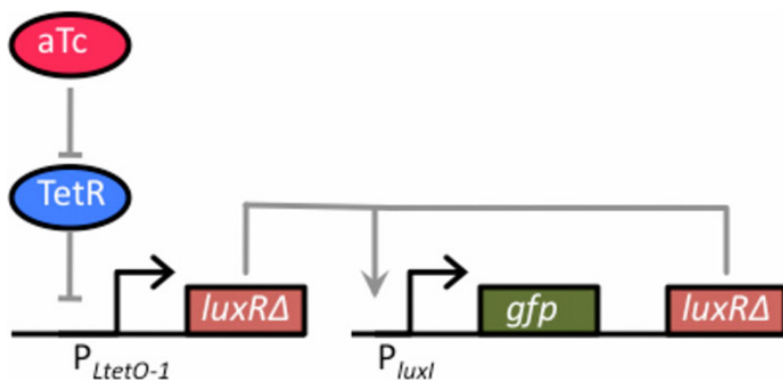

Figure 3 Schematic of tetracycline sensor coupled to the positive-feedback amplifier. The one-component tetracycline sensor consists of a plasmid where $\operatorname{LuXR}_{\Delta 2-162}$ has been cloned behind the TetR-regulated $\mathrm{P}_{\text {Lteto-1 }}$ promoter. In the absence of the inducer anhydrotetracycline (aTc), dimeric TetR binds to the $\mathrm{O} 2$ operator sites within the $\mathrm{P}_{\text {LtetO-1 }}$ promoter and represses transcription. However, when bound with aTc, TetR is no longer able to bind to the $\mathrm{O} 2$ operator sites within the promoter, thus enabling dose-dependent control of $\mathrm{LuxR}_{\Delta 2-162}$. This sensor was coupled with the positive feedback amplifier, encoded on a separate plasmid, by transforming cells (GN100) constitutively expressing a chromosomal copy of the tet $R$ gene with the two plasmids respectively harboring the sensor and amplifier.

chromosomal copy of the tetR gene with the two plasmids respectively harboring the sensor and amplifier (see Materials and Methods for details). A schematic of the integrated design is given in Figure 3. When we tested this design, we found that the amplifier increased both the sensitivity and dynamic range of the integrated circuit relative to an otherwise identical circuit lacking positive feedback (Figure 4). In particular, we found that positive feedback increased the sensitivity to aTc by roughly two orders of magnitude. In other words, we observed equivalent levels of expression in the circuit involving positive feedback at aTc concentrations roughly one hundred times less than those observed with the circuit lacking positive feedback. Moreover, we found that positive feedback increased the dynamic range by roughly $50 \%$. By range, we mean the ratio of expression under saturating inducing (100 $\mathrm{ng} / \mathrm{ml} \mathrm{aTc})$ and non-inducing $(0 \mathrm{ng} / \mathrm{ml} \mathrm{aTc})$ conditions.

In addition to these endpoint measurements, we also performed kinetic experiments where we measured the response over a twelve-hour interval to varying concentrations of aTc (Figure 5). Consistent with our endpoint measurements, we found that the design involving positive feedback was more sensitive to aTc and had a wider dynamic range of expression levels. Collectively, these results demonstrate that our genetic amplifier is capable of both increasing the sensitivity and dynamic range of this one-component tetracycline sensor.

We last tested whether the amplifier would endow the cell with memory. While not a design goal,

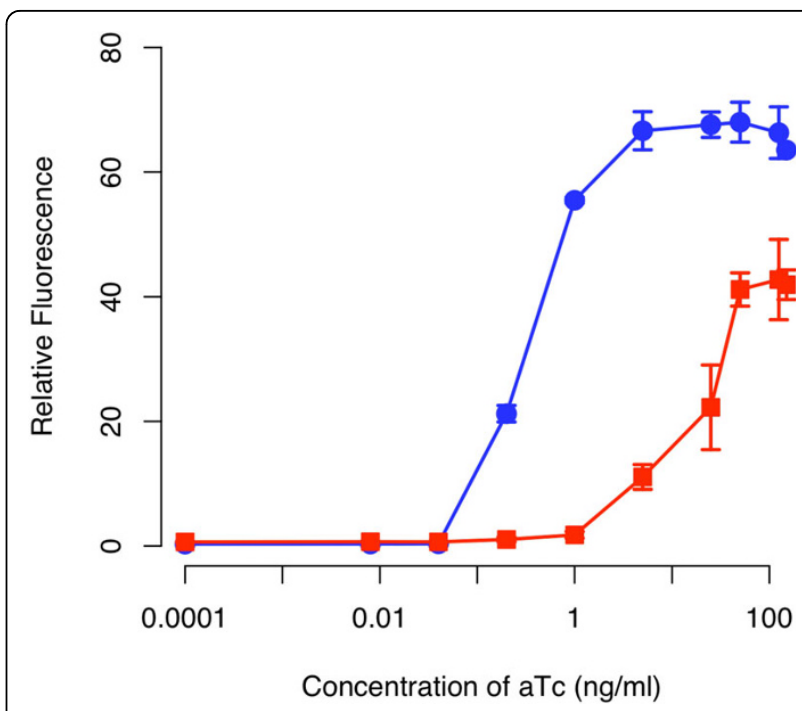

Figure 4 Comparison of tetracycline sensor with positive feedback (solid circles) and without (solid square). Schematic of positive feedback design is shown in Figure 3. The design lacking positive feedback is otherwise identical to one with positive feedback except that only GFP is expressed from the $P_{\text {/uxl }}$ promoter. In these experiments, cells were grown overnight at the indicated concentrations of aTc prior to measurements. The fluorescence values were normalized with the $\mathrm{OD}_{600}$ absorbance to account for cell density. Error bars denote 95\% confidence intervals for the measurement average.

multiple studies have shown that positive feedback can lead to bistability and hysteresis [1,5,23]. Therefore, we speculated that cells harboring the amplifier might be able to "remember" previous exposures to aTc. However, when we transferred cells from media containing aTc to media lacking it, we no longer observed any GFP expression relative to the background after we grew the cells up (data not shown). These results indicate the positive feedback loop involving $\operatorname{LuxR}_{\Delta 2-162}$ is able to amplify the response to an inducer but is incapable of sustaining the response in the absence of inducer.

Based on what we know about the properties of LuxR, specifically the role of AHL in stabilizing LuxR, the reason the circuit does not sustain activation is likely due to the protein dimer being degraded too quickly. In other words, we suspect that $\operatorname{LuxR}_{\Delta 2-162}$ dimer is being degraded at a rate greater than it is being produced by positive feedback alone (though we did not directly make this measurement). More specifically, positive feedback alone is unable to sustain the expression of LuxR $R_{\Delta 2-162}$ in the absence of some exogenous source, in our case the one-component sensor. That said, the positive feedback is still strong enough to amplify the response when an external input signal is present. 


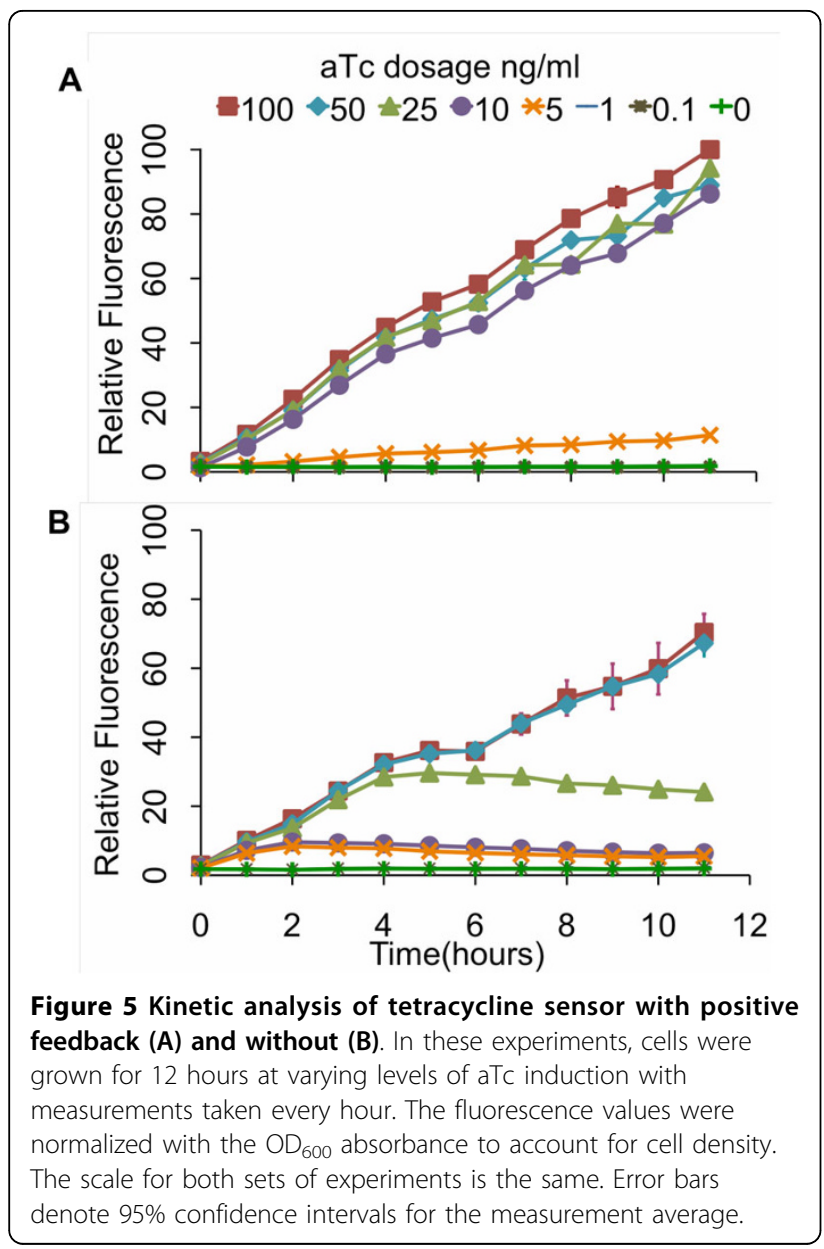

Validation of amplifier using an aspartate sensor

We next tested the amplifier by coupling it to a twocomponent aspartate sensor (Figure 6). To do this, we used the hybrid Tar-EnvZ (Taz) sensor kinase [24]. This chimeric, transmembrane sensor kinase controls the levels of phosphorylated OmpR, which in turn activates the expression from the $\mathrm{P}_{o m p C}$ promoter. When the Taz sensor kinase is bound with aspartate, it increases the levels of OmpR-P, leading to increased expression from the $\mathrm{P}_{\text {omp }}$ promoter. In addition to amino acids, EnvZ chimeras have been constructed to sense other inputs such as sugars and light $[25,26]$.

In order to couple the two-component aspartate sensor with our genetic amplifier, we cloned $\operatorname{LuxR}_{\Delta 2-162}$ behind $\mathrm{P}_{\text {omp }}$ promoter on a compatible, medium copynumber plasmid (p15A origin of replication). To introduce the Taz sensor kinase into $E$. coli, we cloned this gene behind the constitutive $\mathrm{P}_{\text {LtetO-1 }}$ promoter on a compatible, low copy-number plasmid (pSC101 origin of replication). Note, these experiments were performed in cells lacking a chromosomal copy of the $t e t R$ gene, so the $\mathrm{P}_{\text {LtetO-1 }}$ promoter in this background is constitutive.

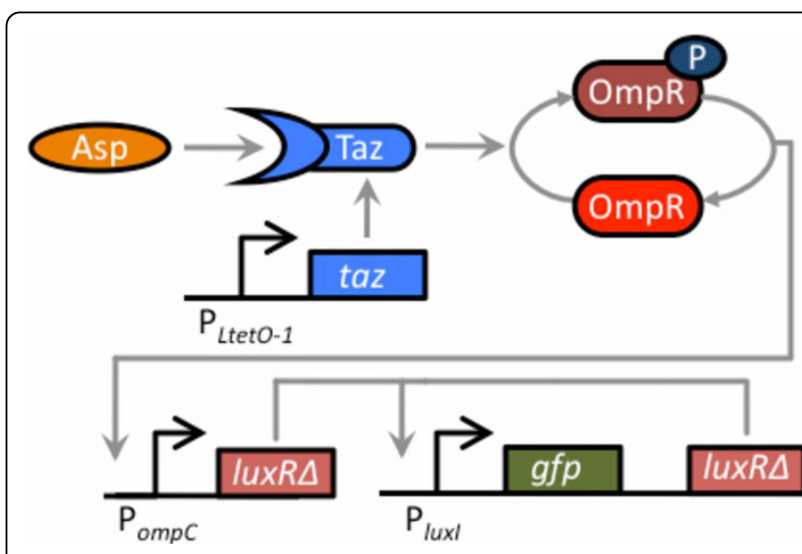

Figure 6 Schematic of aspartate sensor coupled to the positive-feedback amplifier. The two-component sensor consists of the Taz sensor kinase and the OmpR response regulator. Taz controls the level of phosphorylated OmpR (OmpR-P), which in turn activates the expression from the $\mathrm{P}_{\text {ompc }}$ promoter. When the Taz sensor kinase is bound with aspartate, it increases the levels of OmpR-P, leading to increased expression from the $\mathrm{P}_{\text {ompc }}$ promoter. In our design, the Taz sensor kinase has been cloned behind the constitutive $\mathrm{P}_{\text {LtetO-1 }}$ promoter on one plasmid (the cells used in these experiments do not possess TetR). On a second plasmid, LuxR $_{\triangle 2-162}$ has been cloned behind the $\mathrm{P}_{\text {ompc }}$ promoter, resulting in the expression of $\operatorname{LuxR}_{\Delta 2-162}$ being aspartate dependent. The third plasmid harbors the positive feedback amplifier. The sensor was coupled to the amplifier by transforming the three plasmids into a $\triangle$ envZ null mutant (GN101).

To construct the integrated circuit in E. coli, we transformed a $\Delta e n v Z$ null mutant (GN101) with these three plasmids.

Similar to what we observed with the one-component tetracycline receptor, we found that the amplifier increased both the range and sensitivity when coupled to the two-component aspartate sensor as compared to an otherwise identical circuit lacking positive feedback (Figure 7). Unlike the case with the one-component sensor, we observed only a minor increase in sensitivity. However, we observed a significant amplification of the response. In particular, the amplifier increased the dynamic range by roughly an order of magnitude whereas the sensitivity increased by approximately a factor of two. While these results demonstrate that the amplifier is modular as it can readily be applied to different sensor systems, they also demonstrate that the performance of the amplifier is context dependent. In particular, we observed mostly an increase in the range when the amplifier was coupled to the two-component aspartate sensor kinase and, conversely, mostly an increase in sensitivity when it was coupled to the onecomponent tetracycline sensor.

We note that we observed only weak activation, roughly two-fold, in response to aspartate in the absence of positive feedback. This level of activation is less than 


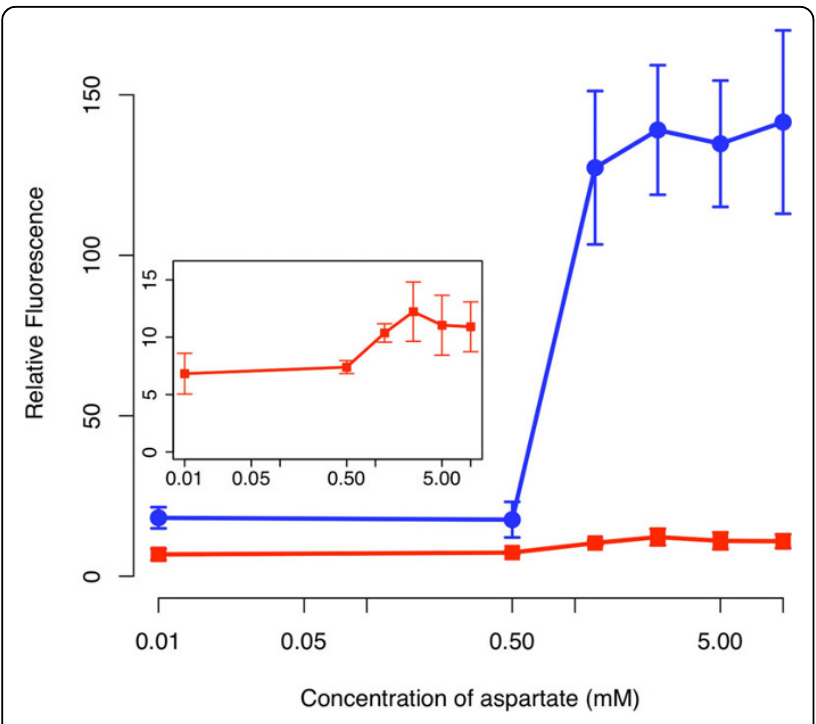

Figure 7 Comparison of sensor output in the presence (solid circles) and absence (solid squares) of the positive feedback amplifier. Schematic of positive feedback design is shown in Figure 6. The design lacking positive feedback is otherwise identical to one with positive feedback except that only GFP is expressed from the $P_{\text {luxl }}$ promoter. In these experiments, cells were grown overnight at the indicated concentrations of aspartate prior to measurements. The fluorescence values were normalized with the $\mathrm{OD}_{600}$ absorbance to account for cell density. Inset figure shows the magnification of the response for the design lacking positive feedback. Error bars denote 95\% confidence intervals for measured averages.

what has been previously observed in other studies using Taz, where the degree of activation is greater than ten fold $[27,28]$. However, unlike our design, these studies measured the expression from the $\mathrm{P}_{\text {omp }}$ promoter. In the present work, we measured the expression from a downstream promoter, $\mathrm{P}_{l u x}$. Thus, there is an additional stage between the sensor and reporter in our design. Likely, expression of $\operatorname{LuxR}_{\triangle 2-162}$ from the $\mathrm{P}_{\text {отр }}$ promoter is not sufficiently strong to activate the $\mathrm{P}_{l u x I}$ promoter without further amplification. However, when we add amplification by including positive feedback, we then obtain robust expression.

\section{Conclusions}

In this work, we developed a simple modular genetic amplifier based on a constitutively active variant of LuxR. We tested this amplifier by coupling it to a one-component tetracycline sensor and a two-component aspartate sensor. In both instances, the amplifier was able to increase the dynamic range and sensitivity of the integrated circuit. Based on these results, this amplifier most likely can be coupled to any cell-based sensor where the output involves the transcription of a gene. In these regards, we have successfully constructed a reusable component.
In addition to sensing applications, the amplifier can also be used to create devices of greater complexity in function. One intriguing application concerns impedance matching. Impedance mismatch occurs when the output range of one sub-circuit does not match the input range of another sub-circuit to which it is connected. To effectively link these two sub-circuits, the respective output and input ranges should match one another. As positive feedback can significantly alter the response of a sub-circuit, it can be used as an impedance matching' device by coupling two different subcircuit circuits together that have disparate requirements for signal levels to operate correctly.

A primary goal of synthetic biology is to design modular components with defined behavior that can be reused in diverse applications [29-32]. The ideal component should have predicable behavior regardless of the context in which it is applied. This is a significant challenge. Even in our experiments, while we rightly hypothesized that we would see amplification due to the positive feedback, we see a different response when we coupled the amplifier to the two different sensors. For instance, the tetracycline sensor showed a major increase in sensitivity but only moderate increase in the dynamic response. The aspartate sensor, however, showed a major increase in the dynamic response but only a moderate increase in sensitivity. Moreover, the amplifier increased background expression in the case of the aspartate sensor but not in the case of the tetracycline sensor. The origins of these differences are unknown, but may arise due to variations, for example, in plasmid copy number, promoter strengths, and the metabolic burden imposed by each circuit. While further engineering can be used to control for these individual factors, their effects are often non-trivial to isolate and quantify.

\section{Acknowledgements}

We thank Lingchong You and Christopher Voigt for plasmids. This work was supported by National Science Foundation grants 0644744 (to CR) and 0943386 (to KB), as well as start up funds provided by the University of Illinois (to KB).

\section{Author details}

'Department of Agricultural and Biological Engineering, University of Illinois at Urbana-Champaign, 1304 W Pennsylvania Ave, Urbana, IL, 61801, USA. ${ }^{2}$ Department of Chemical and Biomolecular Engineering, University of Illinois at Urbana-Champaign, 600 S Mathews Ave, Urbana, IL, 61801, USA.

\section{Authors' contributions}

$\mathrm{CR}$ and $\mathrm{KB}$ conceived the experiments. GN and KW performed experiments. $\mathrm{CR}$ and $\mathrm{KB}$ wrote the manuscript. All authors read and approved the final manuscript.

\section{Competing interests}

The authors declare that they have no competing interests.

Received: 7 September 2009 Accepted: 26 February 2010 Published: 26 February 2010 


\section{References}

1. Mitrophanov AY, Groisman EA: Positive feedback in cellular control systems. Bioessays 2008, 30:542-555.

2. Onsum MD, Rao CV: Calling heads from tails: the role of mathematical modeling in understanding cell polarization. Curr Opin Cell Biol 2009 21:74-81.

3. Novak B, Tyson JJ: Design principles of biochemical oscillators. Nat Rev Mol Cell Biol 2008, 9:981-991.

4. Becskei A, Seraphin B, Serrano L: Positive feedback in eukaryotic gene networks: cell differentiation by graded to binary response conversion. Embo J 2001, 20:2528-2535.

5. Kramer BP, Fussenegger M: Hysteresis in a synthetic mammalian gene network. Proc Natl Acad Sci USA 2005, 102:9517-9522.

6. Maeda YT, Sano M: Regulatory dynamics of synthetic gene networks with positive feedback. J Mol Biol 2006, 359:1107-1124.

7. Ajo-Franklin CM, Drubin DA, Eskin JA, Gee EP, Landgraf D, Phillips I, Silver PA: Rational design of memory in eukaryotic cells. Genes Dev 2007, 21:2271-2276.

8. Stricker J, Cookson S, Bennett MR, Mather WH, Tsimring LS, Hasty J: A fast, robust and tunable synthetic gene oscillator. Nature 2008, 456:516-519.

9. Sayut DJ, Niu Y, Sun L: Construction and engineering of positive feedback loops. ACS Chem Biol 2006, 1:692-696.

10. Sayut DJ, Kambam PK, Sun L: Noise and kinetics of LuxR positive feedback loops. Biochem Biophys Res Commun 2007, 363:667-673.

11. Fuqua C, Greenberg EP: Listening in on bacteria: acyl-homoserine lactone signalling. Nat Rev Mol Cell Biol 2002, 3:685-695.

12. Datsenko KA, Wanner BL: One-step inactivation of chromosomal genes in Escherichia coli K-12 using PCR products. Proc Natl Acad Sci USA 2000, 97:6640-6645.

13. Lutz $\mathrm{R}, \mathrm{Bujard} \mathrm{H}$ : Independent and tight regulation of transcriptional units in Escherichia coli via the LacR/O, the TetR/O and AraC/I1-I2 regulatory elements. Nucleic Acids Res 1997, 25:1203-1210.

14. You L, Cox RS, Weiss R, Arnold FH: Programmed population control by cell-cell communication and regulated killing. Nature 2004, 428:868-871.

15. Miller WG, Leveau JH, Lindow SE: Improved gfp and inaZ broad-hostrange promoter-probe vectors. Mol Plant Microbe Interact 2000, 13:1243-1250

16. Stevens AM, Dolan KM, Greenberg EP: Synergistic binding of the Vibrio fischeri LuxR transcriptional activator domain and RNA polymerase to the lux promoter region. Proc Natl Acad Sci USA 1994, 91:12619-12623.

17. Smith C, Song H, You L: Signal discrimination by differential regulation of protein stability in quorum sensing. J Mol Biol 2008, 382:1290-1297.

18. Urbanowski ML, Lostroh CP, Greenberg EP: Reversible acyl-homoserine lactone binding to purified Vibrio fischeri LuxR protein. J Bacteriol 2004, 186:631-637.

19. Zhu J, Winans SC: The quorum-sensing transcriptional regulator TraR requires its cognate signaling ligand for protein folding, protease resistance, and dimerization. Proc Natl Acad Sci USA 2001, 98:1507-1512.

20. Poellinger KA, Lee JP, Parales JV Jr, Greenberg EP: Intragenic suppression of a luxR mutation: characterization of an autoinducer-independent LuxR. FEMS Microbiol Lett 1995, 129:97-101.

21. Choi SH, Greenberg EP: The C-terminal region of the Vibrio fischeri LuxR protein contains an inducer-independent lux gene activating domain Proc Natl Acad Sci USA 1991, 88:11115-11119.

22. Choi SH, Greenberg EP: Genetic dissection of DNA binding and luminescence gene activation by the Vibrio fischeri LuxR protein. J Bacteriol 1992, 174:4064-4069.

23. Yao G, Lee TJ, Mori S, Nevins JR, You L: A bistable Rb-E2F switch underlies the restriction point. Nature cell biology 2008, 10:476-482.

24. Utsumi R, Brissette RE, Rampersaud A, Forst SA, Oosawa K, Inouye M: Activation of bacterial porin gene expression by a chimeric signal transducer in response to aspartate. Science 1989, 245:1246-1249.

25. Baumgartner JW, Kim C, Brissette RE, Inouye M, Park C, Hazelbauer GL: Transmembrane signalling by a hybrid protein: communication from the domain of chemoreceptor Trg that recognizes sugar-binding proteins to the kinase/phosphatase domain of osmosensor EnvZ. J Bacteriol 1994 176:1157-1163.

26. Levskaya A, Chevalier AA, Tabor JJ, Simpson ZB, Lavery LA, Levy M, Davidson EA, Scouras A, Ellington AD, Marcotte EM, Voigt CA: Synthetic biology: engineering Escherichia coli to see light. Nature 2005, 438:441-442
27. Michalodimitrakis KM, Sourjik $V$, Serrano L: Plasticity in amino acid sensing of the chimeric receptor Taz. Mol Microbiol 2005, 58:257-266.

28. Yoshida T, Phadtare S, Inouye M: The design and development of Tar-EnvZ chimeric receptors. Methods Enzymol 2007, 423:166-183.

29. Bhalerao KD: Synthetic gene networks: the next wave in biotechnology? Trends Biotechnol 2009, 27:368-374.

30. Canton B, Labno A, Endy D: Refinement and standardization of synthetic biological parts and devices. Nat Biotechnol 2008, 26:787-793.

31. Endy D: Foundations for engineering biology. Nature 2005, 438:449-453.

32. Lucks JB, Qi L, Whitaker WR, Arkin AP: Toward scalable parts families for predictable design of biological circuits. Curr Opin Microbiol 2008, 11:567-573.

33. Jin $T$, Inouye $M$ : Ligand binding to the receptor domain regulates the ratio of kinase to phosphatase activities of the signaling domain of the hybrid Escherichia coli transmembrane receptor, Taz1. J Mol Biol 1993, 232:484-492.

doi:10.1186/1754-1611-4-4

Cite this article as: Nistala et al: A modular positive feedback-based gene amplifier. Journal of Biological Engineering 2010 4:4

\section{Submit your next manuscript to BioMed Central and take full advantage of:}

- Convenient online submission

- Thorough peer review

- No space constraints or color figure charges

- Immediate publication on acceptance

- Inclusion in PubMed, CAS, Scopus and Google Scholar

- Research which is freely available for redistribution
C Biomed Central 